\title{
A Comparative Study on Removal of Toxic Mercury and Chromium Using Synthesized Inorganic Complex (WOx-EDA) and Compound (AA) Through a Batch Adsorption Process
}

\author{
M. O. Itodo ${ }^{1} \quad$ U. Lawal ${ }^{2}$ \\ 1.Department of Chemistry, Federal University of Agriculture Makurdi. Nigeria \\ 2.Department of Chemical Sciences, Federal University Wukari. Nigeria
}

\begin{abstract}
Toxic metals ions are well known undesirable pollutant in drinking water. Since they are barely biodegradable and can be accumulated in human body through the food chain to induce a severe threat to human health; it is of great importance to develop low cost, safe and highly effective adsorbents for the adsorptive removal of those toxic metals. Synthesis and Characterization of Inorganic-organic hybrid Tungsten oxide-ethylenediamine (WO $x$-EDA) nanowires have been carried out by a simple, low-cost and high-yield solvo-thermal method. Activated Alumina was also synthesized using direct method. Both Tungsten Oxide ethylenediamine and Activated Alumina (AA) were characterized using, Fourier Transform Infrared (FT-IR) spectroscopy, Scanning Electron Microscopy (SEM) and Transmission Electron Microscopy (TEM). The synthesized inorganic adsorbents adsorption capacities for removing mercury and chromium were investigated using batch adsorption process. The equilibrium data were applied to various sorption kinectic models. The adsorbents adsorptive capabilities were compared. The adsorbents displayed exceptional adsorptive properties; however, WOx-EDA proved to be better adsorbent than activated alumina in terms of removal efficiency. This could be linked to hybrid structure integrated in the functionality of ethylenediamine with the stability of the WOx frameworks. The nanowire morphology and abundant functional amino groups possibly endowed the complex with versatile abilities and highly adsorptive properties.
\end{abstract}

Keywords: Adsorption, Toxic, alumina, Tungsten Oxide Ethylenediamine, Kinetic.

DOI: $10.7176 / \mathrm{CMR} / 11-5-03$

Publication date:May $31^{\text {st }} 2019$

\subsection{Introduction}

It has been particularly interesting to develop an adsorbent demonstrating a high adsorption capacity and low cost for removing various pollutants from contaminated waters. Inorganic chemical adsorbents have thus been studied widely, aiming at providing an alternative to the activated carbon in the treatment of surface and ground water and industrial effluents that contains toxic metal. Some metals are toxic when they form poisonous soluble compounds. Certain metals have no role biologically and are toxic when in a certain forms. Toxic metals can be removed from human system through complexation, ion exchange, chelation and adsorption (Jia Qian and Ashekuzzaman, 2012). Adsorption is a process that occurs when an adsorbate accumulates on the surface on an adsorbent, forming a molecular or atomic film. Adsorption is operative in most natural physical, biological and chemical systems (Gottipati, and Mishra 2012) and (Khattri,et al., 2009). Adsorbents are usually used in granular form; it can vary in size from roughly $10 \mathrm{~mm}$ in diameter to as small as $50 \mathrm{~nm}$. Important properties of adsorbents are adsorption capacity, density, specific surface area, porosity, selectivity and catalytic properties. Adsorbent porosity is the ratio of the volume of the pores and capillaries within the particle to the total volume of the particle (Buekens and Zyaykina, 2001).

Activated Alumina is an inorganic substance that is produced by the dehydration of aluminium hydroxide at high temperature. The material is highly porous and exhibits good surface area, resulting in superior adsorbent capabilities. It does not shrink, swell, soften or disintegrate when immersed in water and it is resistant to thermal shock and abrasion. Alumina possesses amphoteric properties allowing it to act as, either a base or an acid. The ability to alter its particle size or pores provides it with a spectrum of unique biological and physical properties that can address specific desiccant and /or separating needs. One of the important features of activated alumina is that it will bond with other substances without changing its chemistry or form (Tahi et al. 1998). A variety of one-dimensional nanostructured inorganic-organic materials have been reported, such as $\mathrm{CoSe}_{2}$ /amine nanobelts, $\mathrm{ZnS} /$ amine nanowire, GeOx/ethylenediamine nanowires, $\mathrm{Zn}_{2} \mathrm{GeO}$ /ethylenediamine nanoribbons, $\mathrm{ZnSe}$ (diethylenetriamine) nanobelts, and $\mathrm{WO}_{2}$ (deferoxamine) $\left(\mathrm{C}_{7} \mathrm{H}_{6} \mathrm{O}\right)$ nanowires (Polleux et al., 2010). In the novel inorganic-organic hybrid GeOx/EDA (EDA 1/4 ethylenediamine), nanowires with a sub-nanometer periodic structure were fabricated in a high yield via an $\mathrm{Fe}_{2} \mathrm{O}_{3}$-assisted liquid-phase hydrothermal method. An $\mathrm{Fe}_{2} \mathrm{O}_{3}$-assisted mechanism was proposed for the anisotropic growth of the hybrid nanowires. This provides opportunities for the development of new nanomaterials for future applications in electromagnetics, optoelectronics, and sensing technology. The synthesis of $\mathrm{GeO}_{\mathrm{x}} / \mathrm{EDA}$ nanowires was carried out by directly 
mixing $\mathrm{GeO}_{2}$ with $\mathrm{Fe}_{2} \mathrm{O}_{3}$ and then treating the mixture in an aqueous EDA solution at $200{ }^{0} \mathrm{C}$ for 5 days. This aqueous-phase procedure is convenient and has potential for large-scale manufacturing (Gao et al., 2008).

These inorganic-organic hybrid materials combine functional organic components and inorganic building blocks into 1D structure through chemical or physical interactions. More importantly, incorporation of two counterparts may integrate the superior rigidity and thermal stability of the inorganic framework with the structural diversity, flexibility and functionality of organic components, thus synergistically enhancing their performance. However, most studies of these hybrid composites have focused on their electronic and optical properties (Klinke et al.,2005). The advantages of the organic functional groups in adsorption have been largely ignored and there are very few reports that exploit the functional groups of the inorganic-organic hybrid materials for heavy metal ion adsorption ( Zh et al., 2006).

This present study is focused on the synthesis, characterization and the adsorptive properties of Tungsten oxide - ethylenediammine adsorbent (an inorganic functionalized organic hybrid materials) for the aqueous phase detoxification of simulated system containing $\mathrm{Hg}$ and $\mathrm{Cr}$ ions. Sorbent characterization was achieved using FT-IR analysis; Transmission electron microscope, Scanning electron microscope, ultra violet-visible analysis and toxic metals level were quantified using AAS.

\subsection{Materials and Methods}

The reagents for this research include Aluminium chloride, distilled water, sodium hydroxide, mercury salt, chromium salt, ammonium tungstate $\left(\mathrm{NH}_{4}\right)_{10} \mathrm{H}_{2} \mathrm{~W}_{12} \mathrm{O}_{42}$, ethylenediamine and ethanol. All the reagents are of analytical grade, Oven, thermometer, Stopwatch, weighing balance, glass rod, Bunsen burner, Wash bottle, Filter paper, filter funnel, crucible, desiccator, electric furnace, autoclave, conical flask, volumetric flask, beakers, and other routine lab apparatus, FT-Infrared spectrometer (Agilent Technology), Scanning Electron Microscope (MVE016477830), Ultra Visible Spectrometer, transmission electron microscope (Carl Zeiss), Energy dispersive $\mathrm{X}$-ray(Carl Zeiss) are the equipment's used.

\subsection{Synthesis of WOx-EDA hybrid nanowires}

WO $x$-EDA hybrid nanowires were synthesized by a facile solvo-thermal route. Ammonium tungstate $\left(\left(\mathrm{NH}_{4}\right) 10 \mathrm{H}_{2} \mathrm{~W}_{12} \mathrm{O}_{42} \cdot 4 \mathrm{H}_{2} \mathrm{O}, 15.1 \mathrm{~g}\right)$ and ethylenediamine $(60 \mathrm{~mL})$ were mixed under vigorous magnetic stirring for $10 \mathrm{~min}$ and then transferred to a Teflon-lined stainless steel autoclave with an inner volume of $100 \mathrm{~mL}$ and heated to $200{ }^{\circ} \mathrm{C}$ for $24 \mathrm{~h}$, followed by natural cooling to room temperature. The product was collected by centrifugation, washed thoroughly with deionized water (until the $\mathrm{pH}$ reached neutral) and ethanol was used to remove any possible residues and dried in a vacuum oven at $60^{\circ} \mathrm{C}$ overnight (Wei et al., 2014).

\subsection{Synthesis of activated alumina (AA)}

Aluminium chloride $(2.5 \mathrm{~g}$ ) was weighed in a beaker and distilled water of $25 \mathrm{~mL}$ was added and stirred with a glass rod until the solid has dissolved. The solution was warmed to about $40{ }^{\circ} \mathrm{C}$ and, while still stirring with a glass rod, $15 \mathrm{~mL}$ of $2 \mathrm{~mol} \mathrm{dm}^{-3}$ sodium hydroxide solution was added and a white solid was formed. The solid was allowed to settle and a further $1 \mathrm{~mL}$ was added at a time, until the addition of sodium hydroxide solution does not give a white precipitate. A filter funnel with a fluted filter paper was set up. The white mixture was carefully poured into the filter.

Using the wash bottle, the white solid was washed from the beaker until it is completely transferred to the filter paper. When filtration was completed, distilled water was gently squirted over aluminium hydroxide in the filter paper to rinse it. Again, the liquid was allowed to filter away completely. The filter paper was carefully removed from the funnel and placed on a piece of paper slightly larger than the filter paper. It was put in an oven at $105{ }^{\circ} \mathrm{C}$ to dry.

A crucible was weighed; the solid was scraped into the crucible and heated over a small non-luminous Bunsen flame for 10 mins. The crucible and contents was put in a desiccator to cool. The cooled crucible was weighed with the dried aluminum oxide in it. $\mathrm{Al}_{2} \mathrm{O}_{3}$ was activated by heating in an electric furnace at $500{ }^{\circ} \mathrm{C}$ for $24 \mathrm{hrs}$. The activation process was adopted to remove the physically adsorbed species present on the surface of alumina (Hajira and Fahin, 2001).

\subsection{Characterization of synthesized Compounds}

The synthesized adsorbent was characterized using FT-IR for functional groups analysis; SEM analysis for surface morphology, TEM and UV were also used.

\subsection{Preparation of Stock Solution of Metals}

The Hg (II) stock solution was prepared by dissolving $1 \mathrm{~g}$ of $\mathrm{Hg}$ (II) metal in $20 \mathrm{ml}$ of $5 \mathrm{M}$ nitric acid prior to dilution with deionised water to $1 \mathrm{~L}$ volume (Rafeah et al., 2019). The standard stock solution of chromium $(1000 \mathrm{mg} / \mathrm{L})$ was prepared by dissolving $2.828 \mathrm{~g}$ of $99.9 \%$ analytical grade $\mathrm{K}_{2} \mathrm{Cr}_{2} \mathrm{O}_{7}$ in $1000 \mathrm{~mL}$ of distilled 
water. Samples of different concentrations were prepared from this stock solution by appropriate dilutions (Satya et al., 2012).

\subsection{Batch Adsorption Experiment}

Adsorption experiments was based on the method by Song et al.,(2015) was adopted with slight modification in sorbate/sorbent ratio. This experiment was performed to investigate the adsorption characteristics of the two synthesized inorganics. The experiment was carried out by shaking $0.02 \mathrm{~g}$ of adsorbent with $150 \mathrm{~mL}$ of metal ion solution ( $\mathrm{pH}$ 4). This mixture was mechanically shaken for 120 mins. Then, $10 \mathrm{~mL}$ of the solutions was separated from the adsorbents and the residual concentration of heavy metal ion was detected by means of atomic absorption spectroscopy (Song et al., 2015).

\subsubsection{Effect of initial concentration}

The $1000 \mathrm{mg} / \mathrm{L}$ of $\mathrm{Hg}$ and $\mathrm{Cr}$ was diluted to $50,100,150,200$ and $250 \mathrm{mg} / \mathrm{L} .0 .02 \mathrm{~g}$ adsorbents were added to $\mathrm{Hg}$ and $\mathrm{Cr}$ aqueous solutions. The mixture was shaken for $120 \mathrm{mins}$ and the filtrate gotten using centrifuge, and then the metal concentration was analyzed using AAS (Naeema, 2014).

\subsubsection{Effect of $\mathrm{pH}$ on the uptake of $\mathrm{Hg}$ and $\mathrm{Cr}$}

$\mathrm{pH}$ is an important parameter influencing heavy metal adsorption from aqueous Solutions. The effect of $\mathrm{pH}$ on the adsorption capacity of $\mathrm{Hg}$ and $\mathrm{Cr}$ with the synthesized inorganics was examined. The experiment of adsorption as a function of $\mathrm{pH}$ was conducted at initial concentration of $150 \mathrm{mg} / \mathrm{L}$ of $\mathrm{Hg}$ and $\mathrm{Cr}, 0.02 \mathrm{~g}$ of adsorbents at $293 \mathrm{~K}$ (Naeema, 2014)

\subsubsection{Effect of adsorption dosage}

The effect of adsorbent dose on the adsorption process was studied at a contact time 120 minutes for initial $\mathrm{Hg}$ and $\mathrm{Cr}$ concentration of $150 \mathrm{mg} / \mathrm{L}$. The amounts of adsorbents were varied from $0.01,0.02,0.03,0.04$ and $0.05 \mathrm{~g}$ (Naeema, 2014).

\subsubsection{Effect of contact time}

The effect of contact time was studied. The experiment was carried out at an initial concentration of $150 \mathrm{mg} / \mathrm{L}$, the adsorbent dose was $0.03 \mathrm{~g}$, while varying the time (60, 120, 180, 240 and $300 \mathrm{mins}$ ) (Naeema, 2014).

\subsection{Results and Discussions}

3.1 Table 1.0 Physical Characterization of the Synthesized Inorganic Adsorbents

\begin{tabular}{lll}
\hline \multicolumn{1}{c}{ Properties } & Activated Alumina & Tungsten Oxide -Ethylenediamine \\
\hline Colour & White & Yellowish-brown \\
Odour & Odourless & Pungent \\
State & Solid & Solid \\
Percentage Yield & 85.2 & 89.4 \\
Melting Point & $2977^{\circ} \mathrm{C}$ & $3087^{\circ} \mathrm{C}$ \\
\hline
\end{tabular}

\subsection{SEM Characterization}

The surface morphology of the synthesized inorganics were studied using the Scanning Electron Microscope. Figure 1a shows the scanning image of WOx-EDA, while Figure 1b shows the scanning image of AA

The size and shape of the complex was examined using the scanning electron microscope. Figure 1a displays a representative SEM image at 2500 magnification. It revealed that the product is composed of Nano belts with widths and lengths up to several tens of micrometers. Nano belt-like homogeneous morphologies are revealed as flat rocky structures with several holes and black spots showing adsorptive site at 1500 magnification.

The surface morphology of the synthesized activated alumina was obtained using Scanning electron microscope. The SEM image for the synthesized AA was shown in Figure 1b. The SEM image shows a clear surface available for the adsorption. This image also indicated the availability of nano-size pores and cracks on the surface of AA which may be one of the reasons for the significant increase in the adsorption capacity of the synthesized compound. 




Figure 1a: SEM image of Activated Alumina

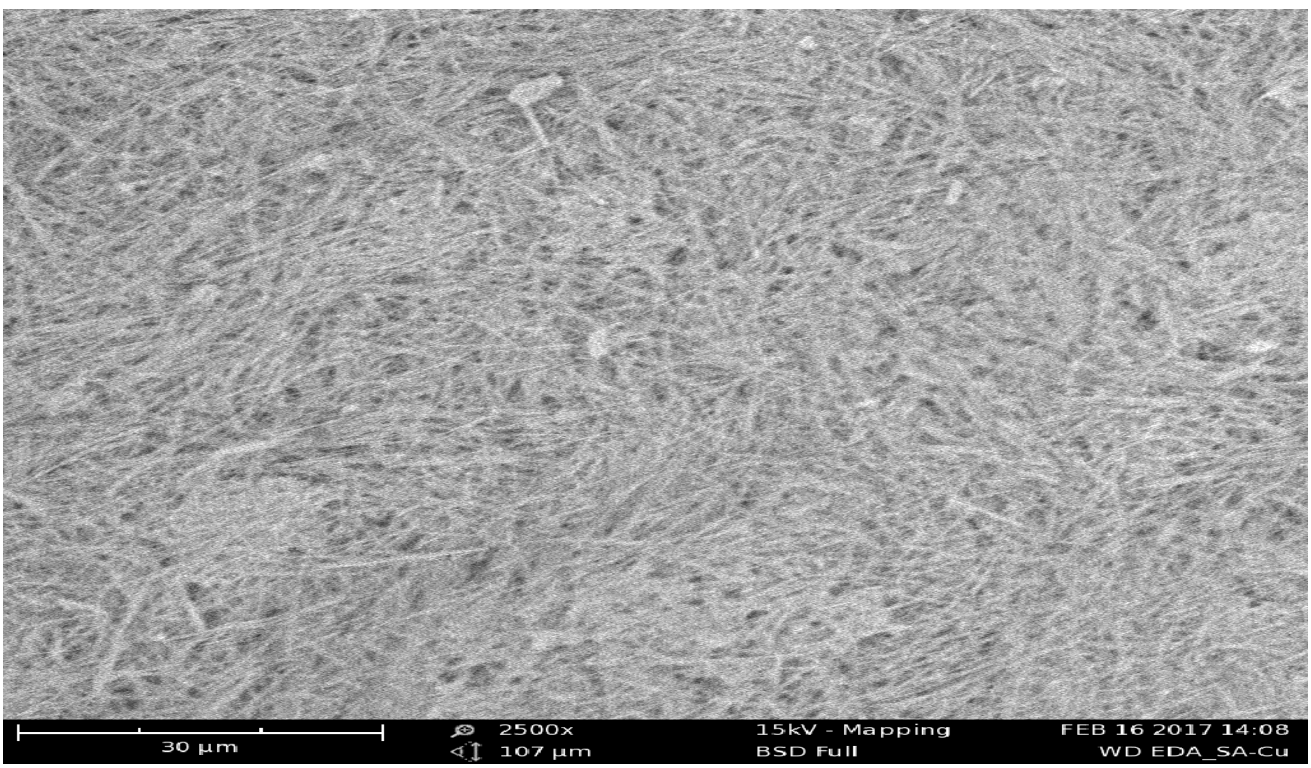

Figure 1b: SEM image of WOx-EDA

\subsection{TEM Characterization of the synthesized inorganics}

The form of WOx- EDA is confirmed by the projected mass-density contrast observed by transmission electron microscopy. TEM image in figure 2a indicates that the WOx-EDA nanowires are straight and uniform. This means their structures have thickness or diameter constrained to tens of nanometers or less and an unconstrained length and also they are composed of repeating molecular units. This was also reported by Xianluo, et al. (2011). The morphology of the synthesized activated alumina in Figure $2 \mathrm{~b}$ however appeared hollow; it portrayed hollow aggregates of lumpy anhedralmacro crystallites. The hollow sites show clearly where Adsorption took place. 


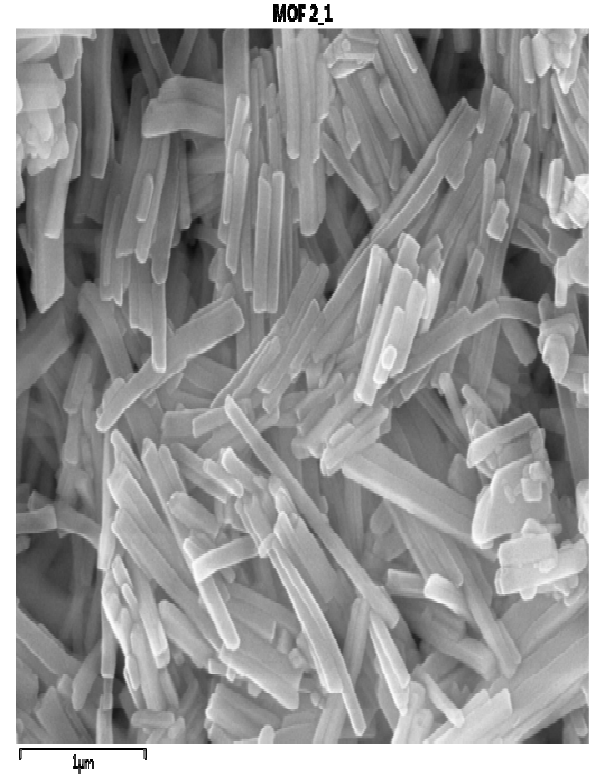

Figure2a: TEM image of WOx-EDA

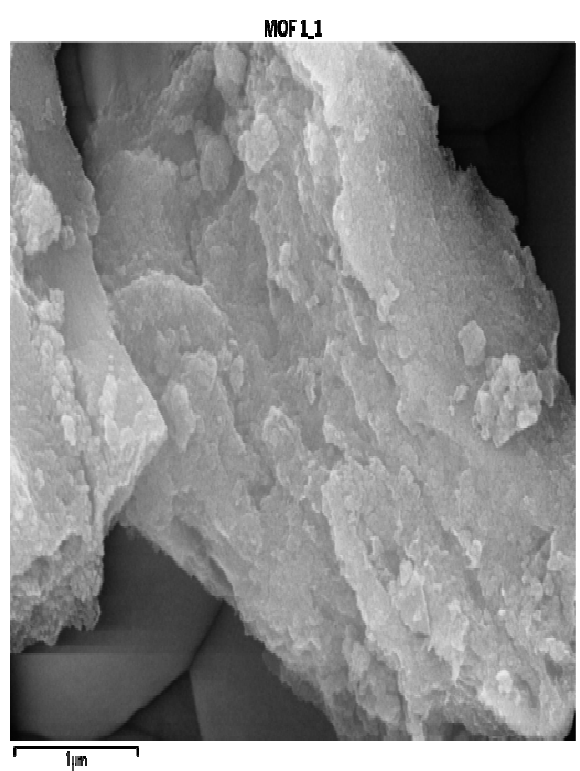

Figure 2b: TEM image of AA

\subsection{UV-visible Spectroscopy characterization of WOx-EDA}

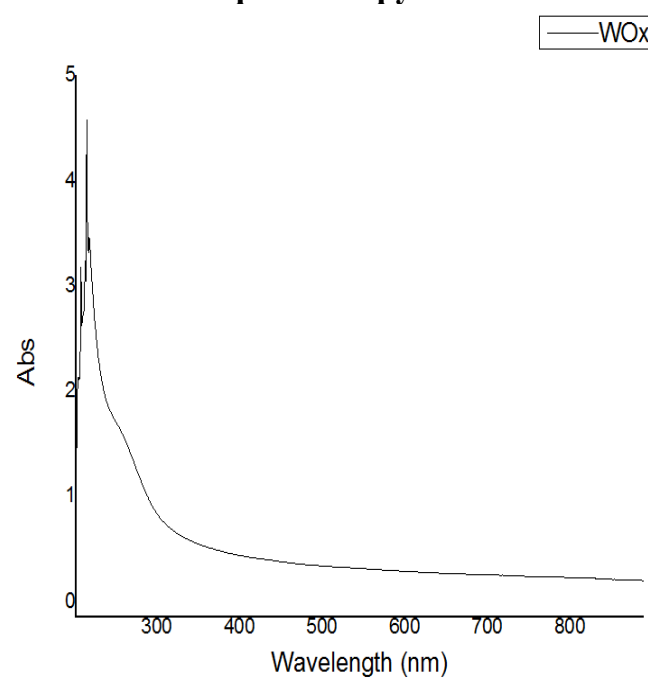

Figure 3a U-V spectrum of WOx-EDAA

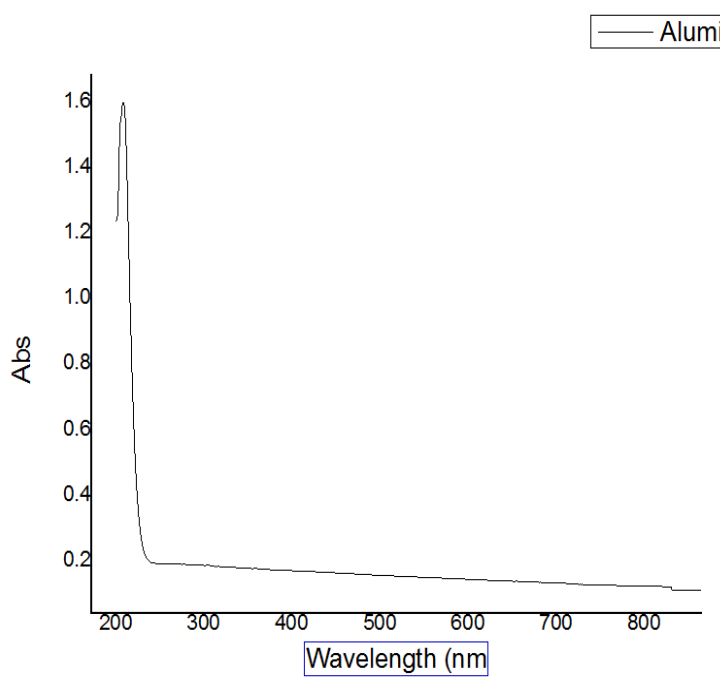

Figure 3b UVspectrum of AA

A sharp band-edge absorption peak at $274 \mathrm{~nm}(4.5 \mathrm{eV})$ was observed in the UV-visible absorption spectrum of the synthesized complex (Figure 3a). The remarkably blue-shifted peak, as compared with conventional tungsten oxide (at 350-450 nm), may be attributed to the strong quantum size effects in these novel hybrid nano belts (ethylenediamine). Incorporation of ethylenediamine molecules, which act as barriers in the inorganic frameworks, could induce strong quantum confinement of both electrons and holes similarly to that reported previously for $\mathrm{ZnTe}(\mathrm{EDA})$ and GeOx/EDA by Xianluo et al. (2011). Figure 3b shows an absorption band around $220 \mathrm{~nm}$ (1.4) this is similar as reported by Lee et al (2015). It reflects the electronic degrees of freedom.

\subsection{FT-IR characterization of WOx-EDA and AA}

The infrared spectra of the WOx-EDA shows a band at $3200 \mathrm{~cm}^{-1}$ which is attributable to $v(\mathrm{O}-\mathrm{H})$ stretching frequencies. The presence of the band at range around 1617.7 indicates the presence of $\mathrm{N}-\mathrm{H}$ bend; this is because of the ethylene diamine ligands. The bands in the spectra of the metal oxide are observed at $408.97 \mathrm{~cm}^{-1}$. The strong bands at 2961.25 and $2895.36 \mathrm{~cm}^{-1}$ are attributable to $v(\mathrm{C}-\mathrm{H})$ stretching frequencies for co-ordination (Wei et al, 2014). From A broad band extending around 511.01 for the AA, is due to symmetric bending of OAl-O (Lee et al., 2015). The band at around $1638.52 \mathrm{~cm}^{-1}$ was equally pronounced in the virgin and in the loaded adsorbents and a slight increase was observed, this could be as a result of absorption that took place on the spent adsorbent, and the band around that region is attributed to bending of $\mathrm{H}-\mathrm{O}-\mathrm{H}$ bonds. The band around 3399.30 $\mathrm{cm}^{-1}$ was also present in all the samples and this could be attributed to the $\mathrm{OH}$ stretching which is likely due to 
the moisture adsorbed on the surface of the compound. Similar work was recorded by Lee et al., (2015).

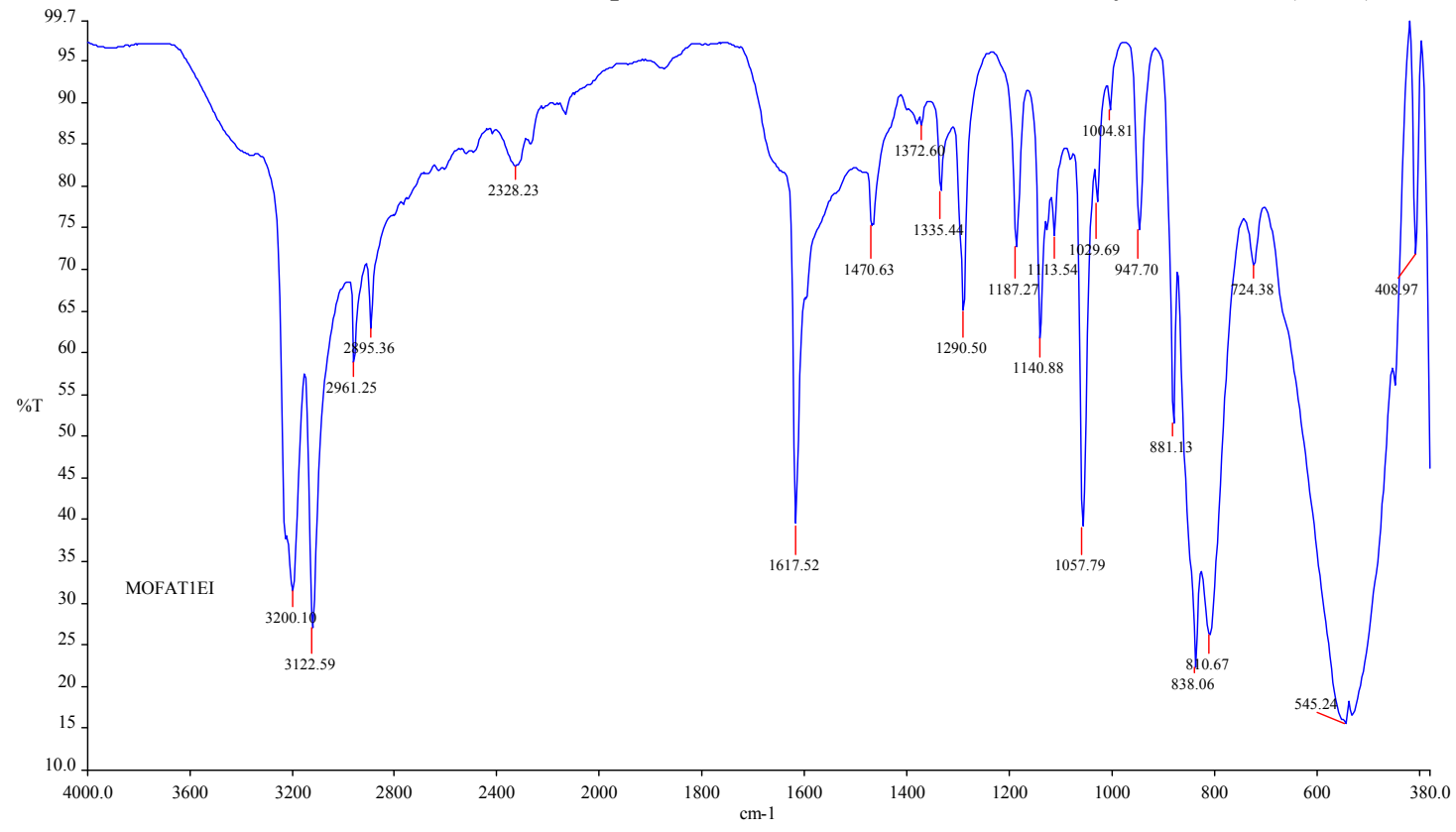

Figure 4a. FT-IR spectral of WOx-EDA

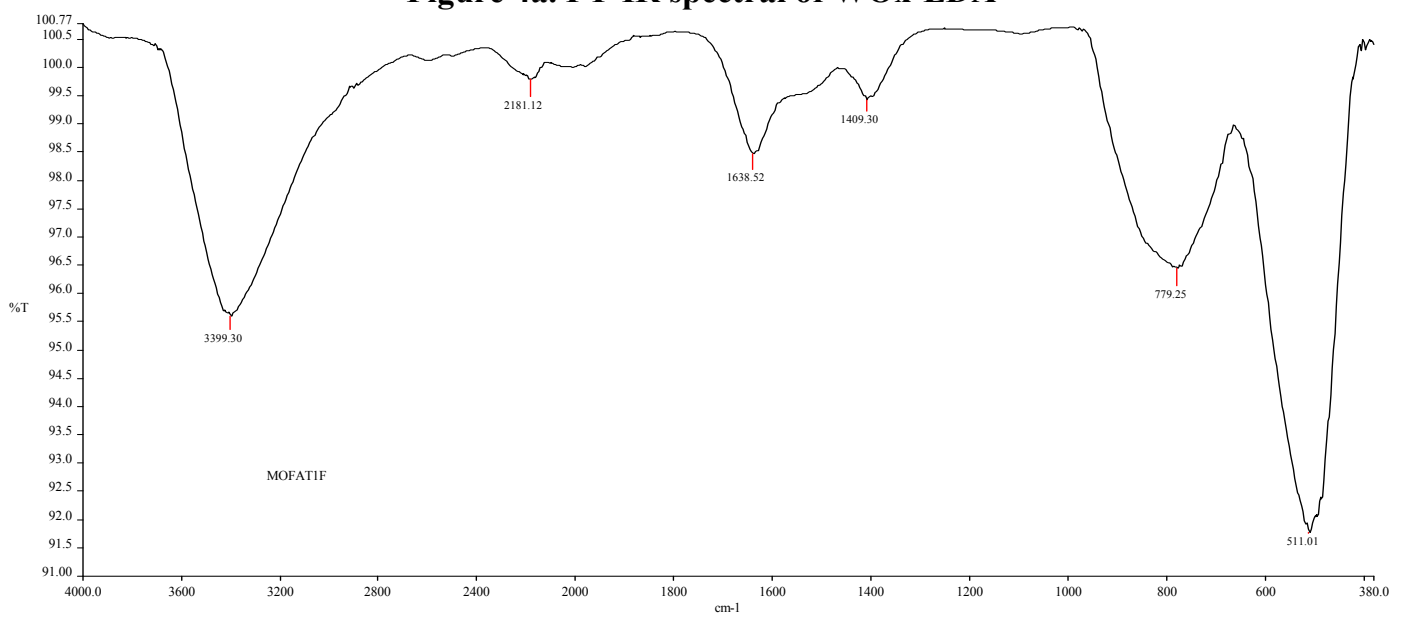

Figure 4b: FT-IR spectral of AA

Table 2.0: FTIR Spectra Characteristics of WOx-EDA

\begin{tabular}{llll}
\hline $\begin{array}{l}\text { Vibrational } \\
\text { Frequency } \\
\left(\mathbf{C m} \mathbf{1}^{\mathbf{1}}\right)\end{array}$ & $\begin{array}{l}\text { Characteristics } \\
\text { Functional } \\
\text { Ganges }\end{array}$ & $\begin{array}{l}\text { WOx-EDA } \\
\left(\mathbf{c m}^{-\mathbf{1}}\right)\end{array}$ & Assignment \\
\hline $\mathbf{3 3 0 0 - 2 5 0 0}$ & O-H Stretch & $3200,3122.5$ & Carboxylic acids \\
$\mathbf{3 0 0 0 - 2 5 0 0}$ & C-H Stretch & 2961.25, & Alkanes \\
& N-H bend & 2895.36 & Amines \\
$\mathbf{1 6 5 0 - 1 5 8 0}$ & C-C stretch (in ring) & 1617.52 & Aromatics \\
$\mathbf{1 5 0 0 - 1 4 0 0}$ & C-N stretch & 1140 & Aliphatic amines \\
$\mathbf{1 2 5 0 - 1 0 2 0}$ & O-H bend & 947 & Carboxylic acids \\
$\mathbf{9 5 0 - 9 1 0}$ & C-H & 881,838 & Aromatics \\
$\mathbf{9 0 0 - 6 7 5}$ & M-O & 488.97 & Metal Oxide \\
\hline $\mathbf{6 1 1 - 4 0 0}$ & & & \\
\hline
\end{tabular}


Table 3.0: FTIR Spectra Characteristics of WOx-EDA

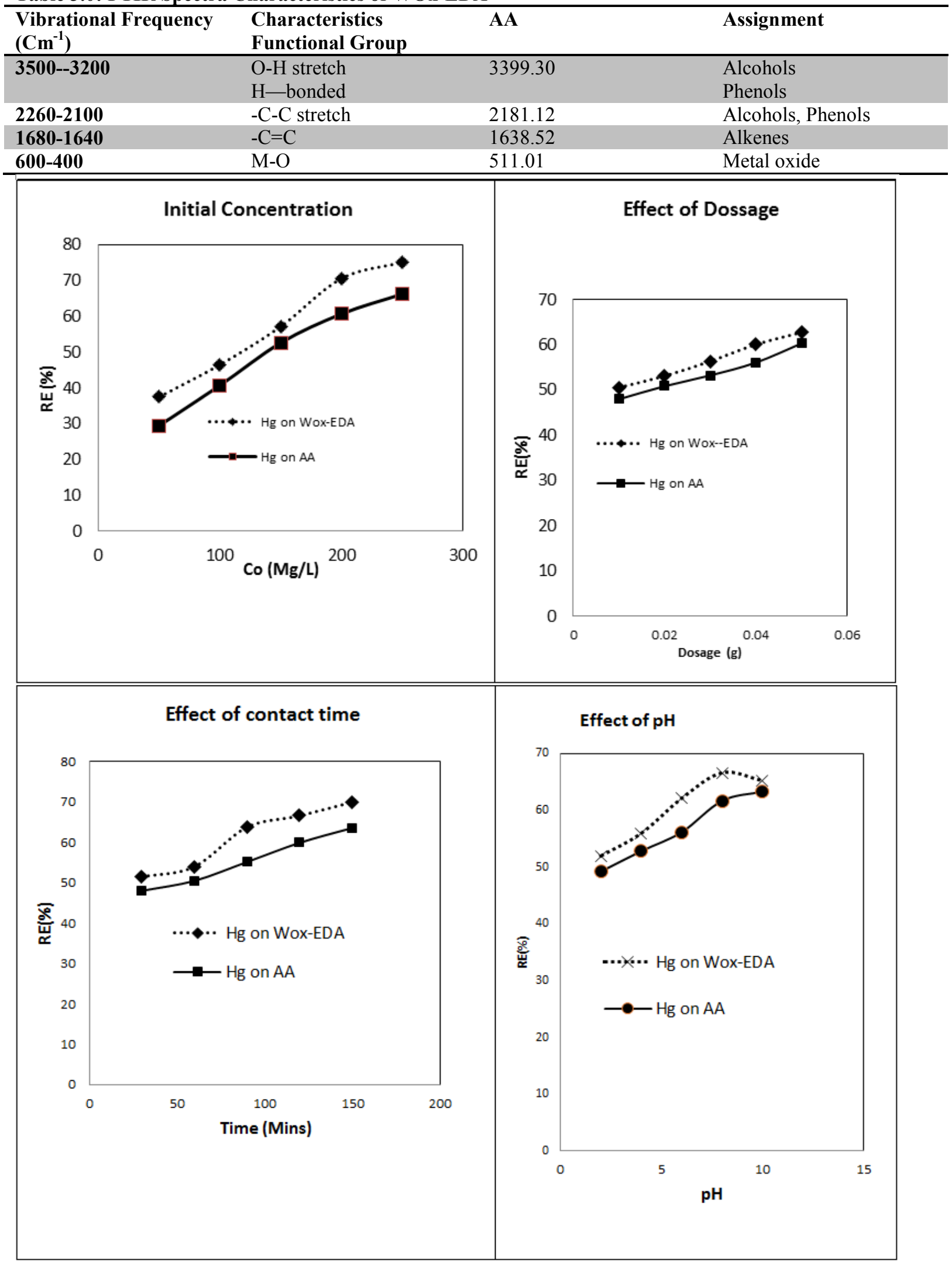

Figure 5a: Comparative Analysis of the Parametric Effects of Hg on WOx-EDA and AA 


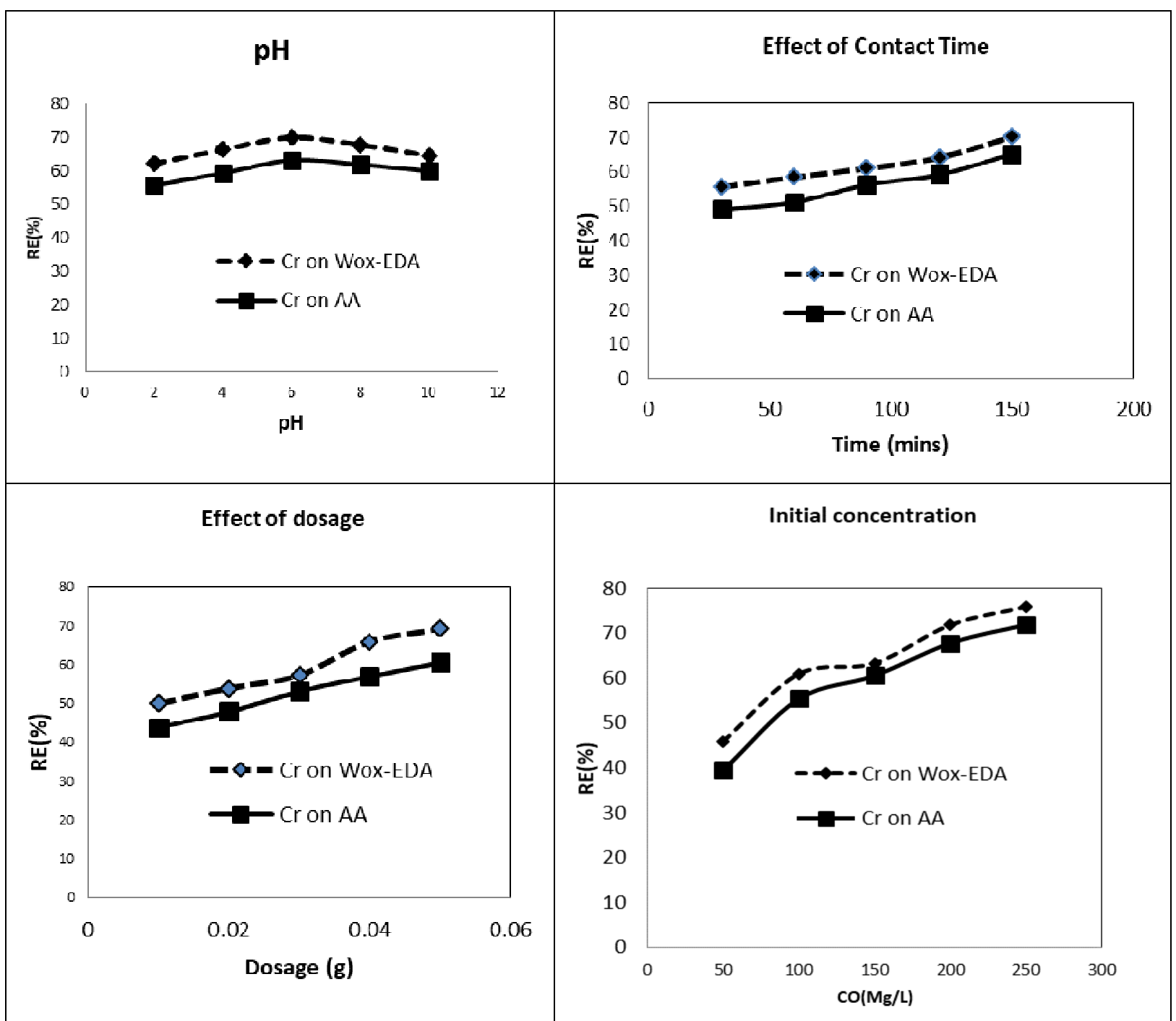

Figure 5b Comparative Analysis of the Parametric Effects of Cr on WOx-EDA and AA

3.6 Batch Adsorption StudiesThe effects of contact time, Adsorbent dosage, temperature and initial metal concentrations were studied. Figure 5 shows the effects of parametric factors on Percentage removal of $\mathrm{Pb}$ and $\mathrm{Cu}$ on AA.

\subsubsection{Effect of Initial Concentration}

For $\mathrm{Hg}$ uptake, the percentage removal increases as the initial concentration of $\mathrm{Hg}$ increases both for WOxEDA and AA with the highest percentage removal found to be $75.11 \%$ at $250 \mathrm{mg} / \mathrm{L}$ for WOx- EDA and $66 \%$ at $250 \mathrm{mg} / / \mathrm{L}$ for AA. Same trend is noticeable in the adsorption of $\mathrm{Cr}$ by WOx-EDA and AA. The highest Percentage removal of $\mathrm{Cr}$ by WOx-EDA and AA are $75 \%$ and $71 \%$ respectively both at $250 \mathrm{mg} / \mathrm{L}$. These results suggest that, if the metal ion concentration in solution increases, the difference in concentration between bulk solution and surface also increases, intensifying the mass transfer processes. Similar result has been reported by investigators Boldizsar et al., (2014).

\subsubsection{Contact Time}

From figure $5 \mathrm{a}$ and $5 \mathrm{~b}$, it was observed that as the contact time increases, the percentage adsorption removal of $\mathrm{Hg}$ and $\mathrm{Cr}$ increased on both WOx-EDA and AA progressively until all the active adsorptive sites have been filled. This is as a result of the availability of a lot of binding sites (Kovo et al., 2015).

\subsubsection{Effect of pH on the removal of $\mathrm{Hg}$ and $\mathrm{Cr}$ by WOx-EDA and AA.}

From the result shown in Figure 5a and 5b. It was seen that the lowest percentage removal was recorded when the $\mathrm{pH}$ was at low $\mathrm{pH}$ for the $\mathrm{Hg}$ and $\mathrm{Cr}$ metal solution on WOx-EDA on AA, and they peaked when at $\mathrm{pH}$ of 6 . When the $\mathrm{pH}$ becomes basic a decrease in the percentage removal was observed. These observations could be because of the higher concentration of $\mathrm{H}^{+}$ion in the solution which competes with the metal solutions in the $\mathrm{pH}$ of 2 and 4 (Wuana et al., 2009). 


\subsubsection{Effect of Adsorbent Dosage}

The effects of varying adsorbent doses of WOx-EDA and AA were investigated for the removal of mercury and Chromium from metal solution. The removal of $\mathrm{Hg}$ by WOx-EDA and AA were found to be highest when the adsorbent dosages were $0.05 \mathrm{~g}$ achieving a percentage removal of $62 \%$ and $60 \%$ respectively. The same trend was noticed in the adsorption of $\mathrm{Cr}$ on WOx-EDA and AA. The increase in removal of $\mathrm{Hg}$ and $\mathrm{Cr}$ with adsorbent dose can be attributed to the introduction of more binding sites for adsorption.

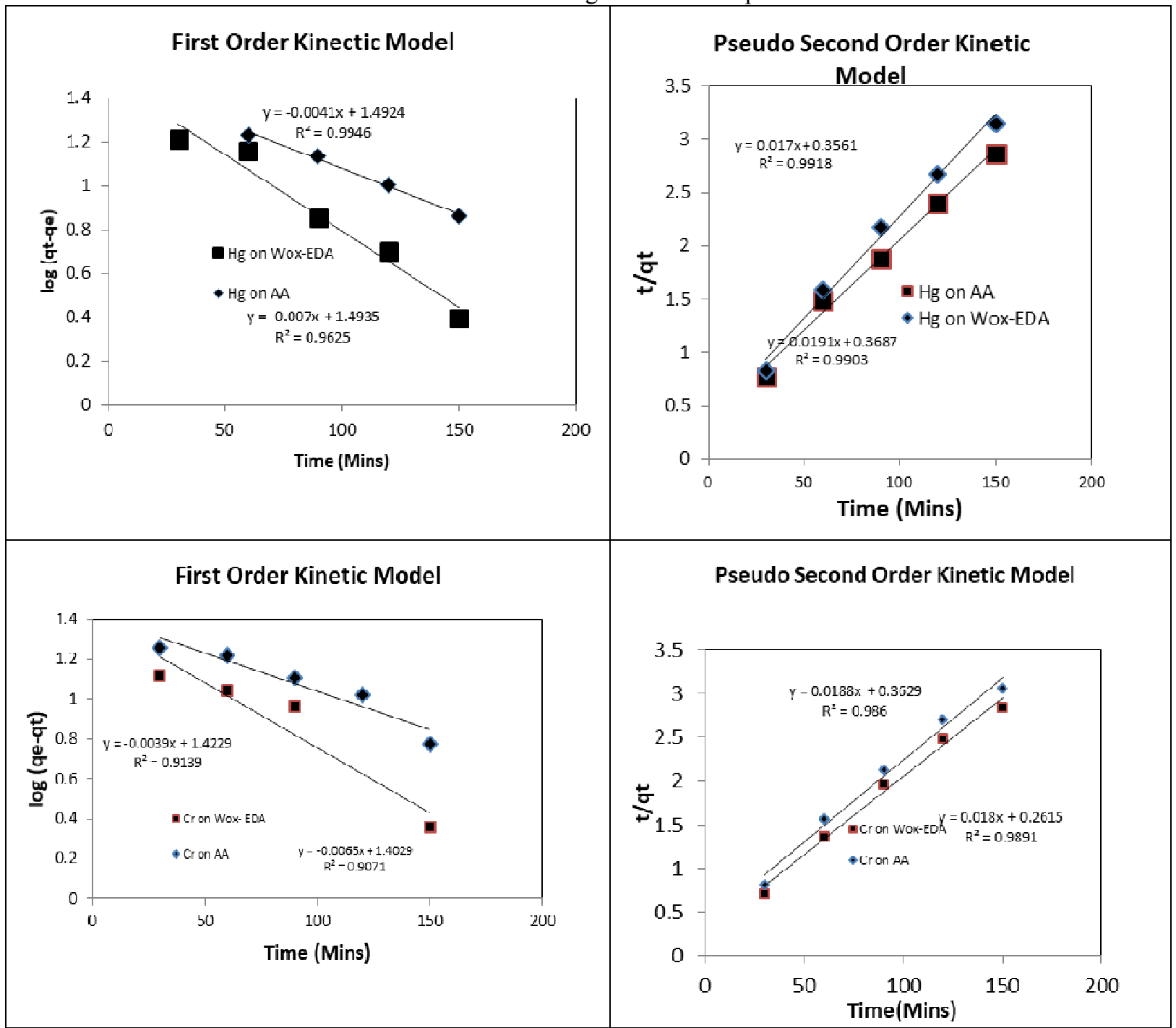

Figure 6: Adsorption Kinetic Plots of Hg and Cr on WOx-EDA and AA

Table 4: Kinetic Studies Experimental Constants for $\mathrm{Hg}$ and $\mathrm{Cr}$ Adsorption onto WOx-EDA and AA

\begin{tabular}{llllll}
\hline Kinetic Model & Constants & WOx-EDA-Hg & AA-Hg & WOx-EDA-Cr & AA-Cr \\
\hline First Order & $\mathrm{R}^{2}$ & 0.96 & 0.99 & 0.90 & 0.91 \\
& $\mathrm{~K}_{1}$ & $-7.0 \times 10^{-3}$ & $-4.1 \times 10^{-3}$ & $-6.5 \times 10^{-3}$ & $-3.9 \times 10^{-3}$ \\
Second Order & $\mathrm{R}^{2}$ & 0.99 & 0.99 & 0.98 & 0.98 \\
& $\mathrm{~K}_{1}$ & $1.9 \times 10^{-2}$ & $1.7 \times 10^{-2}$ & $1.8 \times 10-3$ & $1.8 \times 10^{-3}$ \\
\hline
\end{tabular}

\subsection{Adsorption Kinetics}

The study of adsorption dynamics describes the solute uptake rate and evidently this rate controls the residence time of adsorbate uptake at the solid-solution interface. Taha et al., (2014), in their work, inferred that kinetic models help in the study of adsorption rate, model the process and predict information about adsorbent/adsorbate interaction (physisorption or chemisorption). The kinetics of adsorbates adsorption on the adsorbents were analyzed using first-order and pseudo second-order (Ho and McKay, 1999).

3.7.1 Pseudo first order for adsorption of $\mathrm{Hg}$ and $\mathrm{Cr}$ onto WOx-EDA and AA

The rate constant of adsorption is determined from the first-order equation given by Langergren and Svenska 
(Olugbenga et al., 2010). The $\mathrm{R}^{2}$ values for this model as studied are 0.96 and 0.99 for $\mathrm{Hg}$ onto WOx-AA, while that of $\mathrm{Cr}$ onto WOx-EDA are 0.90 and 0.91 respectively (Table 11). This model considers that the rate of adsorption sites occupation is proportional to the number of unoccupied sites (Blanquez et al., 2011).

\subsubsection{Pseudo second-order kinetic model for $\mathrm{Hg}$ and $\mathrm{Cr}$ onto WOx-EDA and AA}

The coefficient of regression for the pseudo-second order model are 0.999 and 0.99 for $\mathrm{Hg}$ on WOx-EDA and AA respectively and 0.98 and 0.98 for $\mathrm{Cr}$ on WOx-EDA and AA. This suggests the applicability of the pseudosecond order kinetic model to describe the adsorption process. This shows that the overall rate of the adsorption process was controlled by chemisorption and involves valency forces through sharing or exchange of electrons between the sorbent and the sorbate (Ho and McKay, 1999). The second-order kinetics is applicable to the system; since the plot of t/qt versus t, gave a linear relationship (Itodo and Itodo, 2011).

\subsection{Conclusion}

WO $x$-EDA nanowires have been produced via a facile, low-cost, one-pot and scalable solvothermal method using inexpensive raw materials. Activated alumina was successfully synthesized via a simple direct method. This synthesized inorganic affinity for metal ions in simulated waste water was investigated via batch sorption techniques. From the comparative study, it was seen that, Tungsten oxide - ethylenediamine shows better adsorption properties than activated alumina; when parametric effects like initial concentration, dosage, temperature and contact time were varied, this could be as result of the chelation of ligand (which is also a good adsorbent) and hybrid structure that integrates the functionality of ethylenediamine with the stability of the WOx framework. Due to the abundance of amino groups, these WOx-EDA nanowires exhibited high selectivity and affinity for a variety of heavy metal ions and displayed superb adsorption than AA. This capacities for $\mathrm{Pb}^{2+}$ and for $\mathrm{Cu}^{2+}$ Such high adsorption capacities and superior selectivity make WOx-EDA nanowires promising drug in the treatment of metal poisoning. The study also supports the fact that Activated Aluminna absorbent could possibly become a beneficial absorbent for industrial effluents remediation.

\subsection{References}

Blazquez, GM.A. Martin-Lara, G. Tenorio, M. Calero., Batch Biosorption of Lead from Aqueous Solution by Olive Tree Pruning Waste: Equilibrium, Kinetic and Thermodynamic Study. Chemical Engineering Journal, 16 (8):170-177. (2011)

Boldizsar, N. Carmen, M. Andrada, M. Cerasella, I. Barbu-Tudoran, L and Cornelia, M., Linear and Non-linear Regression Analysis for Heavy Metals removal using Agaricusbisporus Macro Fungus. Arabian Journal of Chemistry, 32(2) 554-653. (2014)

Buekens A, Zyaykina N., Adsorbents and Adsorption Process for Pollution Control, Pollution Control Technologies. Vol.II. Encyclopedia of Life Support Systems. (2001)

Gao, Q. S. Chen, P. Zhang, Y. H and Tang, Y., Synthesis and characterization of organic-inorganic hybrid $\mathrm{GeO} x /$ ethylenediamine nanowires. Advance Material, 20(4): 1837-1842. (2008)

Gottipati R, Mishra S., Application of Response Surface Methodology for Optimization of Cr(III) and Cr(VI) adsorption on Commercial Activated Carbons. Research Journal of Chemical Sciences. 2: 40-48. (2012)

Hajira P and Fahin Q., Defluoridation of Water with Activated Alumina : batch operations. Indian journal of Environmental Health, 30 (3) : 262-299. (2001)

Ho, Y.S. and McKay, G., Pseudo-Second Order Model for Sorption Processes .Process Biochemistry, (34): 735 742. (1999)

Hossain M.A, Hao, H. N. Guo W. S and Nguyen T.V., Removal of Copper from Water by Adsorption onto Banana Peel as Bioadsorbent. International Journal of Geomate, 2(4): 227-234. (2012)

Itodo A.U. and Itodo H.U., Application Of Isotherm Studies on the Evaluation of Sorption Energies for Dyestuff Removal onto Derived Thermo-Cracked Shea Nut Shells"; Electronic Journal of Environmental, Agricultural and Food Chemistry. 10 (3): 55:83. (2011)

Jia-QianJiangSMAshekuzzaman Development of novel inorganic adsorbent for water treatment Current Opinion in Chemical Engineering Volume 1, Issue 2, May 2012, Pages 191-199

Khattri S, M. Jekel, M. Singh., Removal of Malachite Green from Dye Wastewater using Neem Sawdust by Adsorption. Journal of Hazardous Material. I67:1089-1094. (2009)

Klinke, C. J. Hannon, L.N. Gignac, K. U. and Reuter, P. J., Tungsten Oxide Nanowire Growth by Chemically Induced Strain, Journal of Physical Chemistry, 10(9): 17787-17790. (2005)

Kovo G. A. Folasegun, A. D and Kayode, O. A. Mechanism on the Sorption of Heavy Metals from BinarySolution by a Low Cost Montmorillonite and its Desorption Potential. Alexandria Engineering Journal, 33 (54):757-767. (2015)

Lee, H.S. Kim, N. Park, T.J. Lee, M. K., Synthesis and Characterization of Metallic Oxides. Chemical Engineering Journal, 2(30): 351-360. (2015)

Mariadas K, G.Kalyani, H.Joga Rao, Y.Prasanna Kumar, P.King The Removal and Equilibrium studies of 
Cadmium by Natural Clay as adsorbent International Journal of Scientific \& Engineering Research, Volume $3: 8,(2012)$

Naeema H.Y., Removal of Toxic Copper Ions uses Alumina. International Journal of Current Microbiology and Applied Science, 4 (77): 415-543. (2014)

Olugbenga B, Oladipo M, Misbaudeen A, Olalekan A., "Kinetic and Equilibrium Studies of Methylene Blue Removal from Aqueous Solution by Adsorption on Treated Sawdust". Macedonian Journal of Chemistry and Chemical Engineering, (29)1: 77-85. (2010)

Oyedeji O. A and Osinfade G. B., Removal of Copper (II), iron (III) and Lead (II) Ions from Mono-component Simulated Waste Effluent by Adsorption on Coconut Husk. African Journal of Environmental Science and Technology. 4(6): 382-387. (2010)

Peery R, D. Green, Perry's Chemical Engineers Handbook, seventh ed., McGraw-Hill, New York, USA, 1999.

Pons, M. P. and Fuste, C. M., Uranium Uptake by Immobilized Cells of Pseudomonas Strain EPS 5028. Applied Microbiology Biotechnology, 3 (9): 661-665. (1993)

Rafeah Wahi, Zainab Ngaini and Veronica Usun Jok Removal of Mercury, Lead and Copper from Aqueous Solution by Activated Carbon of Palm Oil Empty Fruit Bunch World Applied Sciences Journal 5 (Special Issue for Environment): 84-91, 2009. (2009)

Satya V, Yadla1V. Sridevi and .Chandana M. V. Lakshmi., Adsorption Performance Of Fly Ash For The Removal of Lead. International Journal of Engineering Research \& Technology (IJERT) 1(7) 2278-0181

Song He, Chungi Zhao, Peng Y and Supin Y., Synthesis of silicate - supported multidentate ligands Adsorbents for removal of Heavy Metals. Environmental Engineering Science 32 : 593-66. (2012)

Taha M. E, Zeinhom H. M, Walied, S. T and Ahmed M. I., Kinetic and Equilibrium Isotherms Studies of Adsorption of $\mathrm{Pb}$ (II) from Water onto Natural Adsorbent. Journal of Environmental Protection, 7(5) :1667-1681. (2014)

Tahi H, M. Saleem, M. Afzal, H. Ahmad, S.T. Hussain., Removal of Chromium from Tannery Waste using Zeolite-3A. Adsorption Science Technology. 16: 153-161. (1998)

Wei Li, Fang Xia, Jin Qi, Ping Li, Dehong Chen, Zhe Chen, Yu Yu1, Yu Lu, Rachel A. Carus and Weiguo Song Versatile inorganic-organic hybride, Synthesis, mechanism and Application in Waste in Aqueous Solutions: Batch and Fixed-Bed System Studies. Chemical Engineering Journal, 19 (2): 1-7. (2014)

Wuana, R.A. Leke L. Okiebe, D.A and Okei M. N., Aqueous Phase Adsorption of Cu(ii) and Co(ii) Ions from Single and Bisolute Solutions onto Base Treated and Carbonized Rice Husks. Nigerian Journal of Applied Science, 27: 12-136. (2009)

Xianluo H, Qingming J, Jonathan P and Katsuhiko A., Large-Scale Synthesis of WOx-EDA Nanobelts and their Application as Adsorbents. Desalination, 100 (44): 33-61. (2011)

Zh G.T. Zhai, U. B. Gao, X. V. Sheng, Y. F. Wang, H. F. Ma, Y. Z and Yao, J. P., Controllable Assembly of $\mathrm{WO}_{3}$ Nanorods/nanowires into Hierarchical Nanostructures. Journal of Physical Chemistry, 11(30): 238286. (2006) 